\section{Low-voltage CMOS four-quadrant multiplier}

Shen-Iuan Liu and Chen-Chieh Chang

Indexing terms: Analogue multipliers, CMOS integrated circuits, Multiplying circuits

A new CMOS four-quadrant multiplier that can operate from supply voltages of $\pm 1.5 \mathrm{~V}$ is presented. This circuit was fabricated in a standard $0.8 \mathrm{um}$ single-poly double-metal CMOS process. Experimental results show that the nonlinearity can be kept $<2 \%$ across the entire differential input voltage range of $\pm 0.8 \mathrm{~V}$. The total harmonic distortion is $<2 \%$ with the differential input range up to $\pm 0.8 \mathrm{~V}$. The measured $-3 \mathrm{~dB}$ bandwidth of this multiplier is $\sim 5 \mathrm{MHz}$. It is expected to be useful in low-voltage analogue signalprocessing applications.

Introduction: Battery-powered systems such as implantable biomedical systems, portable communication equipment and handheld movie cameras $[1,2]$, etc. require circuits which operate at low supply voltages and have low power consumption. Thus, the demand for analogue circuits that can operate from low supply voltages is very high. Multipliers are a very important building block in many applications, such as adaptive filters, frequency doublers, and modulators. Recently, several low-voltage CMOS/ BICMOS four-quadrant multipliers and amplifiers have been presented using the transistors operated in the triode and weak inversion regions $[3-7]$. In this Letter, a new low-voltage CMOS multiplier is presented. Experimental results are also given to verify theoretical analysis.

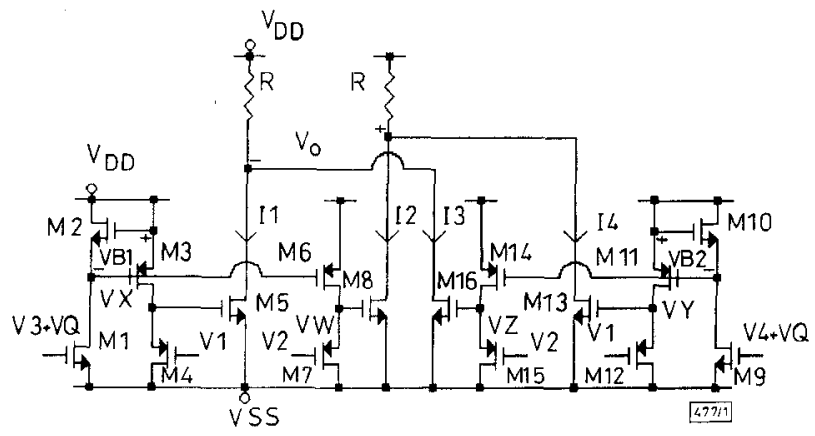

Fig. 1 Proposed low-voltage CMOS four-quadrant multiplier

Circuit description: The proposed four-quadrant multiplier is shown in Fig. 1. Assume that all NMOS transistors in Fig. 1 are matched and operate in the saturation region, as do the PMOS transistors. Neglecting the body effect, the gate-to-source voltages of the NMOS transistors $M_{1}$ and $M_{2}$ will be equal and can be expressed as

$$
V_{Q}+V_{3}-V_{S S}=V_{B 1}
$$

For the matched PMOS transistors $M_{3}$ and $M_{4}$ (also for $M_{6}$ and $M_{7}$ ), a similar relation yields

$$
V_{B 1}=V_{X}-V_{1}=V_{W}-V_{2}
$$

The $n-p$ cross-coupling configuration [8] ensures that $V_{B 1}$ is constant regardless of the types of transistors. For transistors $M_{9}$, $M_{10}, M_{11}, M_{12}, M_{14}$ and $M_{15}$, the following relation can be obtained:

$$
V_{Q}+V_{4}-V_{S S}=V_{B 2}=V_{Y}-V_{1}=V_{Z}-V_{2}
$$

Assume that NMOS transistors $M_{5}, M_{8}, M_{13}$ and $M_{16}$ are matched. Their drain currents can be expressed as

$$
\begin{aligned}
& I_{1}=K\left(V_{X}-V_{S S}-V_{T n}\right)^{2} \\
& I_{2}=K\left(V_{W}-V_{S S}-V_{T n}\right)^{2} \\
& I_{3}=K\left(V_{Z}-V_{S S}-V_{T n}\right)^{2} \\
& I_{4}=K\left(Y_{Y}-V_{S S}-V_{T n}\right)^{2}
\end{aligned}
$$

From eqns. $1-3$, the voltages $V_{X}, V_{Y}, V_{Z}$, and $V_{W}$ can be expressed in terms of voltages $V_{1}, V_{2}, V_{3}, V_{4}, V_{Q}$ and $V_{S S}$. Substituting them into eqns. $4-7$ and after some algebraic calculations, the output current $I_{o}$ of this multiplier can be defined

$$
I_{o} \equiv I_{1}+I_{3}-I_{2}-I_{4}=2 K\left(V_{1}-V_{2}\right)\left(V_{3}-V_{4}\right)
$$

Similarly, the output voltage $V_{o}$ of this multiplier will be

$$
V_{o}=I_{o} R=2 K R\left(V_{1}-V_{2}\right)\left(V_{3}-V_{4}\right)
$$

For proper operation, the following constraints should be satisfied:

$$
V_{S S}-\left|V_{T P}\right|<V_{1}, V_{2}<V_{D D}-\max \left(V_{B 1}, V_{B 2}\right)
$$

and

$$
V_{S S}-V_{Q}+V_{T n}<V_{3}, V_{4}<\left(2 V_{D D}+V_{T n}\right) / 2-V_{Q}
$$

where voltages $V_{T P}$ and $V_{T n}$ are the threshold voltages of the NMOS and PMOS transistors, respectively.

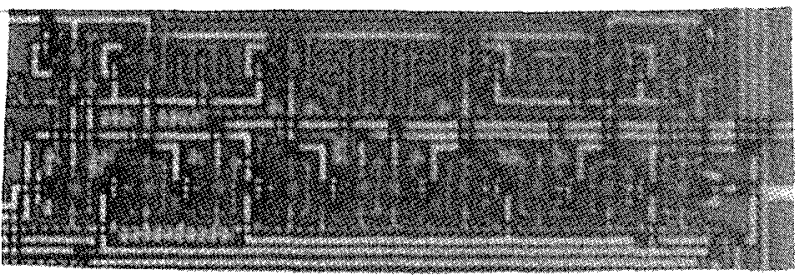

Fig. 2 Die photograph of proposed multiplier

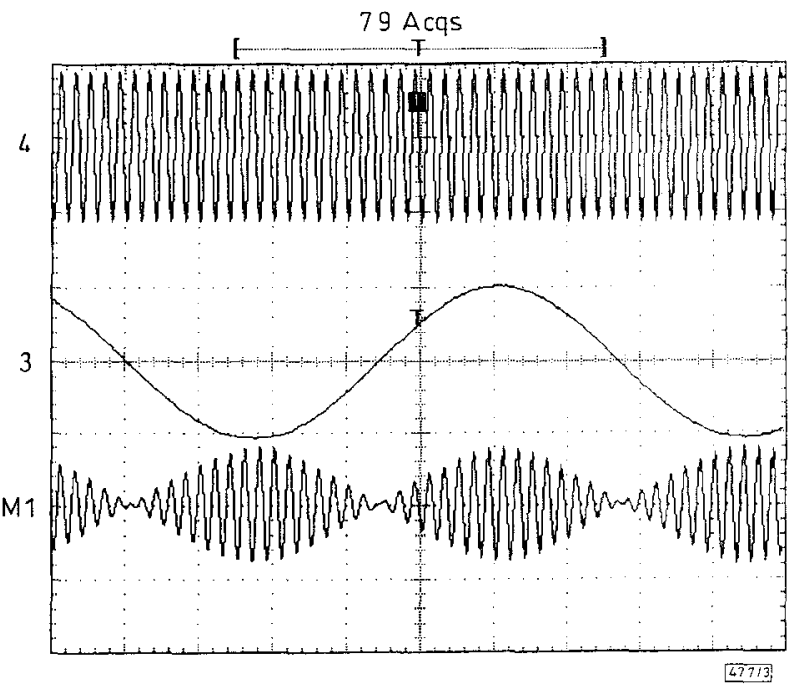

Fig. 3 Output waveforms

Uppermost trace $(500 \mathrm{mV} /$ div $)$ is voltage $\mathrm{V}_{1}\left(=-\mathrm{V}_{2}\right)$ with $0.5 \mathrm{~V}$ $100 \mathrm{kHz}$ sinusoidal signal

Middle trace is voltage $V_{3}\left(=-V_{4}\right)$ with $0.5 \mathrm{~V}, 3 \mathrm{kHz}$ simusoidal signal Lowest trace $(25 \mathrm{mV} / \mathrm{div})$ is output of multiplier Horizontal scale is $50 \mu \mathrm{s} / \mathrm{div}$

Hi Res

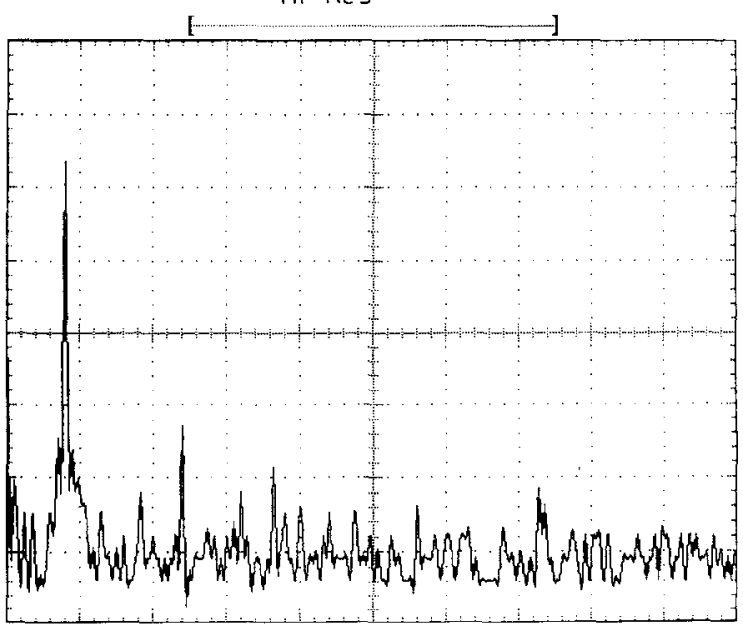

47796

Fig. 4 Measured spectrum of output waveform where $V_{1}\left(=-V_{2}\right)$ is a $0.5 \mathrm{~V}, 20 \mathrm{kHz}$ sinusoidal signal and $V_{3}\left(=-V_{4}\right)=0.4 \mathrm{~V}$

Vertical scale: $10 \mathrm{~dB} / \mathrm{div}$

Horizontal scale: $25 \mathrm{kHz} / \mathrm{div}$ 
Experimental results: The circuit was fabricated in a standard 0.8 $\mu \mathrm{m}$ single-poly double-metal CMOS process. All the aspect ratios $(W / L)$ of the devices in Fig. 1 are equal to $5 \mu / 5 \mu$. The measurement conditions are $R=22 \mathrm{k} \Omega$ and the supply voltage is $\pm 1.5 \mathrm{~V}$. Fig. 2 shows the die photograph of the proposed multiplier. The linearity of the multiplier is within $2 \%$ for $\left|V_{3}\left(=-V_{4}\right)\right|<0.8 \mathrm{~V}$. The measured $-3 \mathrm{~dB}$ bandwidth of this multiplier was $\sim 5 \mathrm{MHz}$. To demonstrate its time-domain response, two signals are applied to the proposed multiplier. The voltage $V_{1}\left(=-V_{2}\right)$ is a $0.5 \mathrm{~V}, 100 \mathrm{kHz}$ sinusoidal signal. The voltage $V_{3}\left(=-V_{4}\right)$ is a $0.5 \mathrm{~V}, 3 \mathrm{kHz}$ sinusoidal signal. Its output waveform is shown in Fig. 3. The total harmonic distortion of the output voltage was found to be $<2 \%$ for $\left|V_{1}\left(=-V_{2}\right)\right|<0.5 \mathrm{~V}$ and $V_{3}=-V_{4}=-0.4 \mathrm{~V}$. Fig. 4 shows the measured spectrum of the output voltage with $V_{1}\left(=-V_{2}\right)$ being a $0.5 \mathrm{~V}$, $20 \mathrm{kHz}$ sinusoidal signal and $V_{3}\left(=-V_{4}\right)=0.4 \mathrm{~V}$.

Conclusions: A low-voltage CMOS four-quadrant multiplier is presented. Experimental results show that the nonlinearity can be kept to $<2 \%$, across the entire differential input voltage range of $\pm 0.8 \mathrm{~V}$. The total harmonic distortion is $<2 \%$, with the differential input range up to $\pm 0.8 \mathrm{~V}$. This multiplier is expected to be useful in many analogue signal-processing applications.

Acknowledgment: The author would like to thank the National Science Council for financial supporting and thank the Chip Implementation Center (CIC), National Science Council, Taiwan, R. O. C. for the fabrication of the test chip. This work was sponsored by NSC-86-2221-E002-056.

\section{(C) IEE 1997}

Electronics Letters Online No: 19970168

22 November 1996

Shen-Iuan Liu and Chen-Chieh Chang (Department of Electrical Engineering, National Taiwan University, Taipei, Taiwan 10664, Republic of China)

\section{References}

1 MEAD, C., and ISMAIL, M.: 'Analog VLSI implementation of neural systems' (Kluwer Academic, Boston, 1989)

2 STOTTS, L.I.: 'Introduction to implantable biomedical IC design', IEEE Circuits Devices Mag., 1989, pp. 12-18

3 RAMIREZ-ANGULO, J.: "Highly linear four-quadrant analog BiCMOS multiplier for $\pm 1.5 \mathrm{~V}$ supply operation'. Proc. IEEE Int. Symp. Circuits and Systems, 1993, pp. 1467-1470

4 WYSZYNSKI, A.: 'Low-voltage CMOS and BiCMOS triode transconductors and integrators with gain-enhanced linearity and output impedance', Electron. Lett., 1994, 30, pp. 211-213

5 COBAN, A.L., and ALLEN, P.E.: 'Low-voltage CMOS transconductance cell based on parallel operation of triode and saturation transconductors', Electron. Lett., 1994, 30, pp. 11241126

6 LIU, S.I.: 'Low voltage CMOS four-quadrant multiplier', Electron. Lett., 1994, 30, pp. 2125-2126

7 LIU, S.I., and CHANG, C.C.: 'CMOS subthreshold four-quadrant multiplier based on unbalanced source-coupled pairs', Int. J. Electron., 1995, 78, (2), pp. 327-332

8 CHENG, M.C.H., and TOUMAZOU, C.: ' 3 V MOS current conveyor cell for VLSI technology', Electron. Lett., 1993, 29, pp. 317-318

\section{Performance estimation of Si/SiGe hetero- CMOS circuits}

\author{
R. Hagelauer, T. Ostermann, U. König, M. Glück and \\ G. Höck
}

Indexing terms: Silicon, Silicon-germanium, CMOS integrated circuits

Semiquantitative performance extrapolation of $\mathrm{Si} / \mathrm{SiGe}$ heterostructure $p^{-}$and $n$-channel devices point to transconductances above $1000 \mathrm{mS} / \mathrm{mm}$ and cutoff frequencies around $200 \mathrm{GHz}$. Circuits such as inverters, logic arrays (e.g. NAND gates) and flip-flops are simulated with feature sizes down to $0.05 \mu \mathrm{m}$ showing a promising performance potential. Delay times of 2.5 and $0.5 \mathrm{ps} / \mathrm{stage}$ are obtained for an inverter chain at a power supply voltage of 1 and $2.5 \mathrm{~V}$, respectively.
Introduction: The progress of microelectronics is based on the advances of CMOS technology. Over the past 20 years, feature sizes have evolved from 6 to $0.25 \mu \mathrm{m}$ and this trend will continue to at least $0.07 \mu \mathrm{m}$. With $0.1 \mu \mathrm{m}$ CMOS technology gate delays of $22 \mathrm{ps} / \mathrm{stage}$ at $1.5 \mathrm{~V} \mathrm{[1]}$ and $11.8 \mathrm{ps} / \mathrm{stage}$ at $2.5 \mathrm{~V}$ [2] have been reported. However, CMOS circuit performance seems to be limited by short channel effects (punch-through, bulk doping, ...) for effective channel lengths $<0.25 \mu \mathrm{m}$. As scaling of silicon becomes more difficult, hetero-FETs (HFETs) or modulation doped FETs (MODFETs) have become of greater interest. Si/SiGe heterostructure technology offers the best opportunity to combine the advantages of heterodevices with the well established Si CMOS technology

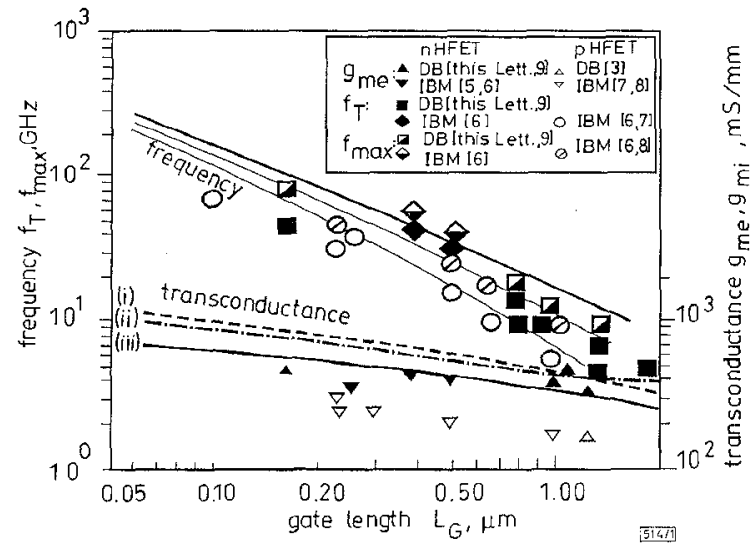

Fig. 1 Gate length dependence of transconductance and cutoff frequencies for SiGe MODFETS

Comparison of experimental data and calculations based on velocity saturation charge control model after Das et al. [10], which was adapted to SiGe HFETs

gml: (i), (ii), (iii) see text

$f_{T}: \stackrel{ }{-} V_{s a l} / 2 \pi L_{G}$ - see text

Si/SiGe hetero-FET performance: $\mathrm{Si} / \mathrm{SiGe}$ hetero-FETs consist of an $\mathrm{Si}$ or $\mathrm{SiGe}$ layer, vertically separated from the MOS or Schottky gate by an SiGe and/or Si layer [3]. The layer sequence can either be undoped or modulation-doped. The 2-D electron or hole gas confined in the channels yield high RT-mobilities, up to $2900 \mathrm{~cm}^{2} / \mathrm{Vs}$ or up to $1800 \mathrm{~cm}^{2} / \mathrm{Vs}$, respectively, at electron or hole concentrations around $1012 \mathrm{~cm}^{-2}[3,4]$ and drift velocities close to the saturation. Preliminary devices have already demonstrated promising performances, e.g. transconductances up to $400-500 \mathrm{mS} /$ mm for $n$-type HFETs and up to $280 \mathrm{mS} / \mathrm{mm}$ for $p$-type HFETs as shown in Fig. 1 [3,5-9] Experiments so far have not established a significant gate length dependence. The simulations for $n-$ HFETs in Fig. 1, based on the velocity saturation charge control model after Das et al. [10] and fitted to the best data at gate lengths at $\sim 1 \mu \mathrm{m}$, assume the channel to be 12 or $20 \mathrm{~nm}$ beneath a Schottky gate, a capacitance of $10^{-6} \mathrm{~F} / \mathrm{cm}^{2}$ (curves (i) and (ii)) or 6 $\times 10^{-7} \mathrm{~F} / \mathrm{cm}^{2}$ (curve (iii)), a mobility of 2500 (curve (i) and (ii)) or $3000 \mathrm{~cm}^{2} /$ Vs (curve (iii)), a drain current constant at $150 \mathrm{~mA} / \mathrm{mm}$ (curve (ii)) or even increasing from 100 to $450 \mathrm{~mA} / \mathrm{mm}$ (curves (i) and (ii)) with decreasing gate lengths $L_{0}$. Transconductances of $>$ $1000 \mathrm{mS} / \mathrm{mm}$ may become possible for $n$-HFETs. For ideal $p$ HFETs, transconductances close to this are expected owing to about the same high mobilities and drift velocities at least when using Ge p-type channels. However, that has not been confirmed by experiments with Ge channels due to technological problems related to the thick SiGe buffer layers and mobilities only at $\sim 500 \mathrm{~cm}^{2} / \mathrm{Vs}[3,7]$. In Fig. I we have also shown cutoff frequencies of $n$ - and $p$-HFETs $[6-8]$ and new own data for $L_{G}=0.18$ to $2 \mu \mathrm{m}$. At around $1 \mu \mathrm{m}, 14 \mathrm{GHz}$ can be obtained and at $0.4 \mu \mathrm{m}$, $\sim 50 \mathrm{GHz}$ for $n$-HFETs. A very high $f_{\max }$ of $81 \mathrm{GHz}$ was recently found for a $0.18 \mu \mathrm{m}$ T-gate $n$-MODFET [9]. The cutoff frequencies of $p$-HFETs are $30-40 \%$ smaller, with a record of $f_{t}=70 \mathrm{GHz}$ at $L_{G}=0.1 \mu \mathrm{m}$ by IBM in collaboration with universities [7]. The frequencies exhibit a clear gate length dependence close to our simulations (based on [10]) with predictions of $>200 \mathrm{GHz}$. The simulations assume a capacitance of $4 \times 10^{-7} \mathrm{~F} / \mathrm{cm}^{2}$, a channel population of $2 \times 10^{12} \mathrm{~cm}^{-2}$, a saturation velocity of $10^{7} \mathrm{~cm} / \mathrm{s}$, no velocity overshoot and mobilities from 1000 to $3000 \mathrm{~cm}^{2} / \mathrm{Vs}$. When the gate to channel distance is increased, the transconductance will 